\title{
Towards Semantic Mapping for Casual Web Users
}

\author{
Colm Conroy \\ Knowledge and Data Engineering Group, \\ Trinity College Dublin \\ coconroy@cs.tcd.ie
}

\section{Introduction}

The Semantic Web approach is becoming established in specific application domains, however there has been as yet no uptake within the mainstream internet environment [1]. The reasons for the lack of uptake of the semantic web amongst casual web users can be attributed to technology perception, comprehensibility and ease of use. It is perceived that the creation of ontologies is a top-down and complex process, whereas in reality ontologies can emerge bottom-up and be simple. Ontology technology is based on formal logics that are not understandable for ordinary people. Finally there is significant overhead for a user in the creation of metadata for information resources in accordance with ontologies. To address these three problems, it is proposed that the interfaces to semantic web tools will need to be engineered in such a way that the tools become simplified, disappear into the background, and become more engaging for casual web users. Increasingly techniques from the semantic desktop research community will enable the creation of a personal ontology on behalf of a user. Although the automatic and efficient matching between the personal ontology and the models used by others (for example through the use of collaborative tags, community ontologies) can be achieved through the application of a variety of matching techniques [2], fully automatic derivation of mappings from the resultant set of candidate matches is considered impossible as yet [3]. A mapping can be thought of as the expression of a confirmed correspondence (e.g. equivalence, subclass, some arbitrary formula). The correspondence could be derived perhaps using machine learning approaches but is typically derived by a human. The majority of state of the art tools in the ontology mapping area [4] and the community ontology creation area [5] rely on a classic presentation of the class hierarchy of two ontologies side by side and some means for the user to express the mappings. These approaches predominately assume that the mapping is being undertaken by an expert: who does not require a personalised interface; whose explicit task is to generate a "one size fits all" full mapping (to be used in common by several applications); and who typically undertakes the task during a small number of long sessions. The number of user trials that have taken place have also been small [6] and those that have, have focused purely on the mapping effectiveness and do not address usability issues (an exception recently being that of [7]). In contrast to the semantic web, 'Web 2.0' has seen an explosion in uptake within the mainstream internet environment [8]. Some of the main characteristics of 'Web 2.0' are rich user experience, user participation and collective intelligence [9]. We intend to take user-driven methodologies that exist 
within 'Web 2.0' to semantic mapping. We propose that the casual web users who will benefit from mappings (through usage by their applications), will undertake themselves partial targeted mappings, gradually and over time, using techniques that address usability issues, support personalization and enable control of the mapping interactions.

\section{State of the Art}

A widespread issue in making semantic mapping, indeed most semantic, tools more accessible to user is the lack of focus on support and usability for users. PROMPT [10] is a plugin for Protégé which supports managing multiple ontologies including ontology merging and mapping. PROMPT presents a mapping suggestion from a candidate list of mappings to the user which the user verifies or rejects then another suggestion is displayed and the cycle is repeated until the user deems the mapping complete. User evaluations for PROMPT were performed in [11] and the results showed that merging was quite difficult for each user and in particular users found performing any non-automated procedures quite difficult. The paper [12] presents a theoretical framework for cognitive support in ontology mapping. It provides some software tool requirements for each framework principle it suggests, e.g. 'reason for suggesting a mapping'. CoGZ is a mapping tool, built upon PROMPT, which supports this framework using TreeMaps [13] to present an overview of the ontologies and possible mappings. A large usability study to refine the cognitive support framework is planned as future work. In [14] the idea of ontology matching is extended to community-driven ontology matching. Their approach was to partition people into multiple different communities (groups) where alignments can be shared, which extends and preserves the advantages given to communities by the web. A prototype was developed based on their approach and showed the feasibility of acquisition and sharing of ontology mappings among web communities. A side effect of their approach is improving the recall of matching tools via repository of ontology mappings for different domains. Investigating the benefits for human contributors is planned as further work. The paper [15] extends S-Match [16] into a semantic matching system which provides proof and explanations for mappings it has discovered. The matching system uses the Inference Web infrastructure [17] to expose meaningful fragments of S-Match proofs in which users can browse. Proofs are displayed using short, natural language, high level explanations without any technical details which are designed to be intuitive and understandable by ordinary users. Results have been promising and shown the potential to scale for the semantic web. In [18] a proposed formal model for ontology mapping creation is suggested. The formal model is used to get complete correspondence between user's actions and generated alignments. They propose a set of different graphical perspectives that can be linked with the same model, each of them offering different viewpoints on the displayed ontology. By using these different perspectives they hope to hide the complexity of the underlying logical language and give better understanding of the mapping action. 


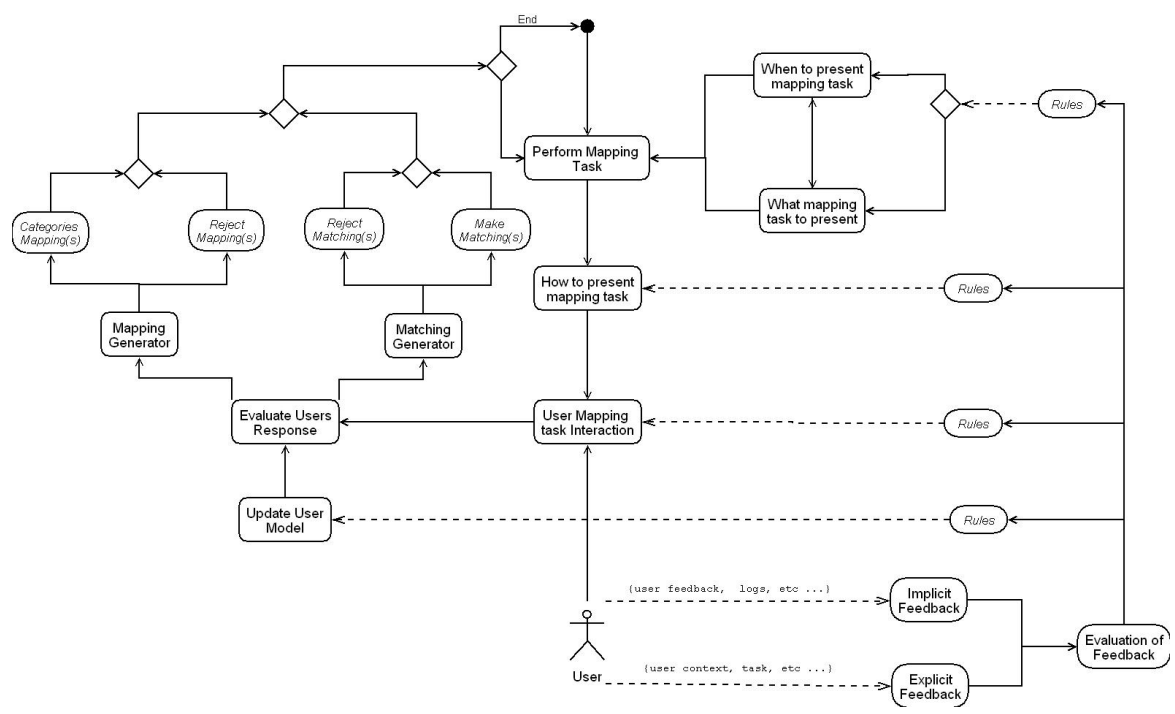

Fig. 1. The Mapping Process for Casual Web Users

\section{Research Question}

The research question to be addressed is: what kind of interactions will be acceptable, efficient and effective for casual web users to achieve semantic mappings gradually and over time between conceptual models of interest to the user. In order to achieve semantic mappings, users will need to undertake mapping tasks as part of the mapping process: e.g. make a mapping based on matching information, categorise a mapping, identify possible mappings, provide corrections for a mapping and possibly withdraw or reject a mapping. Through interaction with the mapping system, the user will undertake the mapping tasks. In our opinion, these interactions need to be adapted so that casual web users see benefit of engaging with the mapping process and are kept usefully engaged over a long period. For example possible available axes of adaptation could be personalisation, visualisation, collaboration, context and the choice of mapping tasks. These adaptations can occur both at runtime or design time. The adaptations can also be either applied to the mapping process or within the system. In this $\mathrm{PhD}$ the primary focus will be on runtime adaptation of interactions for an end-user within a single mapping process based on context. We prioritise the focus on context-based adaptations of interactions due to the lack of research on this topic within the semantic mapping area. Our definition of context is the current browsing environment the user is within i.e. if the user is using a browser, what webpage and task are they engaged in? In particular the work will:

- Develop an adaptive mapping process framework to assist casual web users: There is a need to make the ontology mapping process as unintrusive and as natural as possible, as it is important not to interrupt ordinary users during their daily tasks. This is to ensure that they do not see mapping tasks as inconvenient work but more as something that will be beneficial to them. 
- Determine key context-driven adaptation of interactions with the user and the effect of these: The key factors in this problem are determining the most appropriate contexts for people to engage in the mapping process and the appropriate mapping tasks for these contexts.

- Develop an experimental framework based on the above process for evaluation of adaptive interactions in a long-term mapping process on the web: Such an experimental framework for mapping does not currently exist in the state of the art, as most systems implement "one shot" mapping approaches.

An outcome of this research will be a process (Fig. 1), methodology and tools.

\section{Evaluation}

In this section we outline our current and previous experiments.

\subsection{Natural Language}

In our initial experiment undertaken early in 2007 we aimed to determine the most practical way of visually displaying the mapping information for different groups of users. Our hypothesis was that using a "Question \& Answer" natural language interface to visually display ontological information helps in making mapping more familiar and accessible and also reduces the complexity of the mapping process for users. Our experiment investigated the effect and usability of a natural language prototype tool (NL) [19] on three classes of users and tried to determine whether it made the mapping task more user friendly for one group when compared to the others. In addition we wanted to contrast our tool against a current state of the art mapping tool. We chose COMA++ [4] as our state of the art tree-type graph mapping tool. The three different classes of users were: ontologically aware, technology aware and casual web users. The resulting paper [19] describes the experiment in more detail, some key conclusions drawn were:

- $\quad$ On the positive side, results suggested casual web users can map effectively and efficiently even compared to ontologically aware users. Using Natural Language seemed to help people read and understand the information and the Q\&A approach helped in navigating through the mapping task.

- On the negative side, casual web users found it very restrictive to be limited to a narrow range of mapping terminology, e.g. "corresponds" and "similar to" when answering mapping questions. In addition, some users were unclear about the benefit in engaging in the mapping task.

\section{2 'Tagging' Approach (Finishing August 2008)}

In our current experiment we are focusing on whether it is valuable to embed the mapping process within the user environment, designing a user-centric mapping process, and addressing the negative concerns garnered from the previous experiment 
by allowing the user to be more expressive by allowing them to 'tag' the mapping relation. Our hypothesis is the mapping task can be simplified, become unintrusive and more engaging by using a 'tagging' approach paradigm, embedding the mapping process within the user environment and showing the benefits of the mapping task. By using the power of "Web 2.0" through a Firefox extension [20] within our new 'tagging' prototype, we aim to engage the user and display matching tasks at appropriate times within their own work environment, see [21] for more details. We use online questionnaires, interviews, and a log of each user's actions to evaluate the impact of the 'tagging' prototype. In particular through the use of our implementation over the coming months we aim to investigate whether casual web users will be able to use tagging to turn matches into expressive mappings in a straightforward, practical and natural manner. We will also investigate whether embedding the mapping interface inside a browser extension will allow the mapping process to take place over time within a casual web users' work environment in an unobtrusive, sensible, and normal way. The benefits in engaging in the mapping task for the user will be the gain of individually tailored RSS news feed items.

\section{Next Step: Context-Driven (September 2008 - June 2009)}

A key dilemma is making the mapping process unintrusive yet still engaging to casual web users. In the next experiment we are going to explore what mapping task should be performed given the context of the user. For example, whether only specific mapping tasks should be performed within a certain context, e.g. when on a site such as del.icio.us [22] only mapping tasks specific to this website should be asked like aligning your 'tags' with the del.icio.us domain ontology. A potential benefit of aligning each user model with the domain ontology is allowing the sharing of information between each user, e.g. alignment on the YouTube [23] site allows sharing of video's via friend of a friend while the user is looking at a video which is similar. The mapping task to be performed should be dependent on both the user context and the web task the user is performing, e.g. if the user is writing an email or document no tasks should be performed while if the user is organizing their bookmarks on the del.icio.us then context-driven mapping tasks might be suggested. The infrastructure setup will involve developing an experimental platform which allows for the evaluation of hundreds of users on the web over a long time period. We would hope to discover via this experiment whether a contextdriven mapping task approach will encourage casual web users' to interact with the mapping process.

\section{Conclusions}

Through our evaluation we intend to weigh up what kind of interactions are necessary for casual web users' to achieve semantic mappings in an acceptable, efficient and effective manner. The thesis write up will occur from July 2009 till October 2009. 


\section{References}

1. Berners-Lee, T., Shadbolt, N., Hall, W.: The Semantic Web Revisited. IEEE Intelligent Systems 21(3), 96-101 (2005)

2. Shvaiko, P., Euzenat, J.: A Survey of Schema-based Matching Approaches. J. Data Semantics IV, 146-171 (2004)

3. Noy, N.: Semantic Integration: A Survey of Ontology-Based Approaches. ACM SIGMOD Record 33(4), 65-70 (2004)

4. Aumüller, D., Do, H., Massmann, S., Rahm, E.: Schema and Ontology Matching with COMA++. In: ACM SIGMOD international conference on Management of data, pp. 906908 (2005)

5. Zhdanova, A.: Towards Community-Driven Ontology Matching. In: ACM 3rd International Conference on Knowledge Capture, pp. 221-222 (2005)

6. Jameson, A.: Usability and the Semantic Web. In: Sure, Y., Domingue, J. (eds.) ESWC 2006. LNCS, vol. 4011, p. 3. Springer, Heidelberg (2006)

7. Falconer, S.M., Noy, N.F., Storey, M.: Towards understanding the needs of cognitive support for ontology mapping. In: International Workshop on Ontology Matching, ISWC 2006, CEUR-WS, vol. 225 (2006)

8. Madden, M., Fox, S.: Riding the Waves of 'Web 2.0'. Technical report, Pew Internet \& American Life Project (2006)

9. O'Reilly, T.: What is Web 2.0: Design Patterns and Business Models for the Next Generation of Software. J. Communications \& Strategies 1, 17 (2007)

10. Noy, N., Musen, M.: The PROMPT suite: interactive tools for ontology merging and mapping. J. Human-Computer Studies 59(6), 983-1024 (2003)

11. Lambrix, P., Edberg, A.: Evaluation of ontology merging tools in bioinformatics. In: Pacific Symposium on Biocomputing 2003, pp. 589-600. World Scientific, Singapore (2003)

12. Falconer, S.M., Storey, M.: A Cognitive Support Framework for Ontology Mapping. In: Aberer, K., Choi, K.-S., Noy, N., Allemang, D., Lee, K.-I., Nixon, L., Golbeck, J., Mika, P., Maynard, D., Mizoguchi, R., Schreiber, G., Cudré-Mauroux, P. (eds.) ASWC 2007 and ISWC 2007. LNCS, vol. 4825, pp. 114-127. Springer, Heidelberg (2007)

13. Shneiderman, B.: Tree visualization with tree-maps: 2-d space filling approach. ACM Transaction on Graphics 11(1), 92-99 (1992)

14. Zhdanova, A., Shvaiko, P.: Community-driven ontology matching. In: Sure, Y., Domingue, J. (eds.) ESWC 2006. LNCS, vol. 4011, pp. 34-49. Springer, Heidelberg (2006)

15. Shvaiko, P., Giunchiglia, F., Pinheiro da Silva, P., McGuinness, D.: Web Explanations for Semantic Heterogeneity Discovery. In: Gómez-Pérez, A., Euzenat, J. (eds.) ESWC 2005. LNCS, vol. 3532, pp. 303-317. Springer, Heidelberg (2005)

16. Giunchiglia, F., Shvaiko, P., Yatskevich, M.: S-Match: an algorithm and an implementation of semantic matching. In: Bussler, C.J., Davies, J., Fensel, D., Studer, R. (eds.) ESWS 2004. LNCS, vol. 3053, pp. 61-75. Springer, Heidelberg (2004)

17. McGuinness, D.L., Pinheiro da Silva, P.: Infrastructure for web explanations. In: Fensel, D., Sycara, K.P., Mylopoulos, J. (eds.) ISWC 2003. LNCS, vol. 2870, pp. 113-129. Springer, Heidelberg (2003)

18. Mocan, A., Cimpian, E., Kerrigan, M.: Formal Model for Ontology Mapping Creation. In: Cruz, I., Decker, S., Allemang, D., Preist, C., Schwabe, D., Mika, P., Uschold, M., Aroyo, L.M. (eds.) ISWC 2006. LNCS, vol. 4273, pp. 459-472. Springer, Heidelberg (2006) 
19. Conroy, C., O’Sullivan, D., Lewis, D.: A Tagging Approach to Ontology Mapping. In: 2nd International Workshop on Ontology Mapping, ISWC 2007, CEUR-WS, vol. 304. Springer, Heidelberg (2007)

20. Firefox add-ons, add new functionality to browsing experience, http: / /addons.mozilla.org/

21. Conroy, C., O'Sullivan, D., Lewis, D.: Ontology Mapping Through Tagging. In: International Workshop on Ontology Alignment and Visualisation, CISIS 2008, pp. 886891 (2008) ISBN 0-7695-3109-1

22. Del.Icio.Us, A social bookmarks manager, http://del.icio.us

23. YouTube, user-generated video site, http://www.youtube.com 